\title{
Transarterial Chemoembolization Improves Survival in Advanced Hepatocellular Carcinoma Patients Treated with Tyrosine Kinase Inhibitors Plus Immune Checkpoint Inhibitors
}

Yue Hu ( $\square$ huyue58@126.com )

Third Affiliated Hospital of Sun Yat-Sen University https://orcid.org/0000-0003-2661-1195

\section{Tao Pan}

Third Affiliated Hospital of Sun Yat-Sen University

\section{Xi Cai}

Third Affiliated Hospital of Sun Yat-Sen University

Quansheng He

Third Affiliated Hospital of Sun Yat-Sen University

\section{Yubao Zheng}

Third Affiliated Hospital of Sun Yat-Sen University

\section{Bing $\mathrm{Hu}$}

Third Affiliated Hospital of Sun Yat-Sen University

\section{Ting Jiang}

Third Affiliated Hospital of Sun Yat-Sen University

\section{Mingsheng Huang}

Third Affiliated Hospital of Sun Yat-Sen University

\section{Zaibo Jiang}

Third Affiliated Hospital of Sun Yat-Sen University

Junwei Chen

Third Affiliated Hospital of Sun Yat-Sen University

\section{Chun Wu}

Third Affiliated Hospital of Sun Yat-Sen University

\section{Research Article}

Keywords: Hepatocellular carcinoma (HCC), TACE, Sorafenib, Lenvatinib, Nivolumab, Pembrolizumab, Camrelizumab, Combination therapy, Overall survival, Tumor response, Adverse events

Posted Date: July 8th, 2021 
DOl: https://doi.org/10.21203/rs.3.rs-679376/v1

License: (c) (1) This work is licensed under a Creative Commons Attribution 4.0 International License. Read Full License 


\section{Abstract}

\section{Background}

The survival benefit and safety of transarterial chemoembolization (TACE) for advanced Hepatocellular Carcinoma (HCC) patients treated with tyrosine kinase inhibitors (TKIs) and immune checkpoint inhibitors (ICls) is unclear. We aimed to investigate the efficacy and safety of TACE combined with TKIs and ICls the treatment of advanced HCC.

\section{Methods}

In this study, the conditions of 147 patients with advanced HCC who underwent TKIs plus ICls treatment between July 2017 and April 2020 were evaluated. We divided these patients into the TACE group and non-TACE group based on whether they were treated with TACE during TKIs plus ICls treatment, and compared their survival outcomes, especially overall survival (OS), and whether they were exposed to unexpected toxicities.

Results

In this study, a total of 98 patients who underwent TACE during TKIs plus ICls treatment were included in the TACE group, while the other 49 patients were included in the non-TACE group. According to the Modified Response Evaluation Criteria in Solid Tumors (mRECIST), the objective response rate (ORR) of the TACE group was higher than that of the non-TACE group (ORR $74.5 \%$ vs. $40.8 \%, p \otimes 0.001$ ). The OS of the TACE group was significantly longer than the non-TACE group (OS 19.3 months vs. 10.8 months, $p=$ 0.010 ). The incidence of grade 3-4 toxicities in the TACE group was similar to that in the non-TACE group (33.7\% vs. $28.6 \%, p=0.532)$.

\section{Conclusions}

The TACE treatment combined with TKIs plus ICls resulted in longer OS compared to the treatment of systemic TKIs plus ICls without TACE during the process of advanced HCC.

\section{Introduction}

Hepatocellular Carcinoma (HCC) is the fifth most common cancer worldwide and the second most common cause of cancer-related death [1]. The radical treatment of early HCC includes local resection, liver transplantation and ablation. However, most HCC patients are complicated with more serious cirrhosis or are diagnosed during the advanced stage (unresectable/extrahepatic metastasis) [2]. These patients are no longer suitable for radical treatment. Thus, more effective treatments need to be explored for them.

The systematic treatment of HCC has undergone profound changes in the past decade. Since 2007, the tyrosine kinase inhibitors (TKIs) represented by sorafenib have significantly prolonged survival in patients 
with advanced HCC [3], thus having profoundly improved treatment strategies of HCC. In 2018, clinical trial results demonstrated that the overall survival of Lenvatinib treatment is not inferior to sorafenib and is recommended by several guidelines as the standard of first-line systemic treatment [4-6]. In addition, three other drugs, regorafenib, cabozantinib, and ramucirumab, have been used as second-line therapy since then $[1,7]$. However, the survival improvement of TKIs monotherapy for HCC was not satisfactory. In recent years, a variety of immune checkpoint inhibitors (ICls), such as nivolumab, pembrolizumab, and camrelizumab, successfully demonstrated their effectiveness as a treatment of HCC [8-10]. However, head-to-head clinical trials of ICls and sorafenib failed to improve outcomes [11,12].

TKls can reprogram the immunosuppressive microenvironment around tumors into an immunestimulating environment, in which conditions the use of ICls enhances the antitumor activity of T cells [13]. Currently, several studies have demonstrated the great potential of TKIs combined with ICls in the treatment of HCC. A Phase 1b, single-arm study [14]showed that the median overall survival (OS) of lenvatinib plus pembrolizumab were obviously improved (OS: 22 months, $95 \%$ confidence intervals [Cl]: 20.4 months, not estimable [NE]), which are encouraging signs of antitumor activity observed in HCC patients. The IMbrave150 trial [15] showed that atezolizumab plus bevacizumab delivered a higher survival rate than sorafenib in patients with untreated unresectable HCC (6 months OS $84.8 \%$ vs. $72.2 \%$, $p \llbracket 0.001)$, which is the first further improvement of OS in unresectable HCC patients. The treatment mode of TKIs combined with ICls greatly extended the survival time compared to the TKIs monotherapy. Nevertheless, the tumor response rate remains at a low level even after combination treatment, which is an outcome that may further benefit patients.

Transarterial chemoembolization (TACE), an effective locoregional therapy, has been recommended as one of the common treatments for HCC by most guidelines $[5,16,17]$ In 2020 , TACTICS trial ${ }^{18}$ comparing TACE plus sorafenib with TACE alone reported a major improvement in PFS based on a new definition of untreatable progression, TACE plus TKIs proved to be feasible. Otherwise, TACE can promote immunogenic cell death and induce tumor-associated antigen specific response, thus enhance tumor response to ICls [19]. The combination of TACE with TKIs and ICls is expected to further improve the survival outcome for HCC patients.

So far, there have been few formal reports on TACE combined with TKIs plus ICls for the treatment of advanced HCC, and the efficacy and safety are still uncertain. In this study, we divided the treated patients into two groups according to whether they underwent TACE during the treatment of TKIs plus ICls. Survival outcomes of TACE combined with TKIs plus ICls in advanced HCC patients were compared and observed, as well as any unexpected toxicities.

\section{Materials And Methods}

\section{Study Design}


The Ethics Review Committee has approved this study of The Third Affiliated Hospital of Sun Yat-sen University. Data of patients with advanced HCC who received TKIs plus ICls treatment between July 2017 and April 2020 were respectively collected, and the follow-up period was up to January 2021. As it is a retrospective study, informed consent of patients was waived.

The diagnosis of HCC meets the requirements of the AASLD guidelines [6]. Inclusion criteria for patients were as follows: (a) 18-75 years old, (b) had an Eastern Cooperative Oncology Group (ECOG) performance status of $0-1$, (c) had Child-Pugh class A or B liver disease, and (d) adequate organ function. Patients were excluded from our study if they (a) missed follow-up data, (b) had any prior systemic therapy, (c) had other previous malignant tumors, (d) had no assessable intrahepatic tumor, (e) had hepatic encephalopathy, uncontrolled ascites or pleural effusion.

From July 2017 to April 2020, a total of 147 eligible patients with advanced HCC received TKIs plus ICls, including 98 patients who received TACE during combination therapy (hereafter, TACE group) and 49 who received combination therapy without TACE (hereafter, non-TACE group), as shown in retrospective data compilation (Fig. 1). The TACE treatment was performed according to the evaluation of the attending physician and the patient's willingness. Before treatment, the attending physician fully informed the patients of the possible treatment costs, possible treatment outcomes and treatment-related side effects. All of the included patients expressed understanding regarding the potential effects of the TACE, and therefore made clear treatment choices.

\section{TACE Procedure}

TACE is performed under the guidance of digital subtraction angiography (DSA) by experienced interventional radiologists during endovascular interventional therapy. TACE received 1 week prior to baseline was allowed as intra-follow-up treatment. The chemotherapeutic agents, embolization materials used in TACE, and detailed TACE procedures have been described in previously published articles [20]. Repeat TACE is allowed if the follow-up imaging taken after one month of operation suggests tumor survival and the TACE-induced damaged is tolerated by liver function.

\section{TKIs Management}

Alternative TKls during TKIs plus ICls treatment include sorafenib and lenvatinib. The initial oral dose of sorafenib is $400 \mathrm{mg}$ twice daily, and lenvatinib is $12 \mathrm{mg}$ (if bodyweight $\geq 60 \mathrm{~kg}$ ) or $8 \mathrm{mg}$ (if bodyweight $<60 \mathrm{~kg}$ ) once per day. The initial dose should be maintained and continued if there is no intolerant toxicity or radiographic disease progression. Patients in the TACE group resumed their prescribed dose of TKIs $3-5$ days after TACE. If the adverse events of TKIs are not tolerated at the standard dose, the dose is allowed to be halved to reduce the adverse effects. If the toxic effects are still intolerable with the halved dose, treatment should be suspended until the adverse events are relieved or disappeared.

\section{ICls Management}


During the TKIs plus ICls treatment, the options for ICls include nivolumab, pembrolizumab, and camrelizumab, and the administration of ICls and TKIs start on the same day. These ICls were given intravenously every 3-4 weeks at a standard dose, with each infusion lasting for at least half an hour until disease progression changes or intolerable toxicity associated with ICls appears.

\section{Assessment of Tumor Response and Treatment Safety}

Patients in both groups received abdominal contrast-enhanced CT or MR examination to setup baseline, followed by initial and follow-up exams within 1-3 months and every 1-2 months thereafter until data cutoff, death or loss of follow-up occurs. Response to treatment was assessed according to Response Evaluation Criteria in Solid Tumors, version 1.1 (RECIST1.1) [21]and modified RECIST (mRECIST) [22]. Two radiologists with more than 15 years of experience in abdominal imaging independently evaluate the target lesions, and any inconsistencies in the evaluation results were resolved by consensus. Tumor evaluation indexes include complete response (CR), partial response (PR), stable disease (SD), and progression disease (PD). The ORR includes $C R$ and $P R$, while the disease control rate (DCR) is defined as the sum of $C R, P R$ and $S D$.

Adverse events (AEs) that occurred during the follow-up were evaluated in terms of classification, incidence and severity, according to National Cancer Institute Common Terminology Criteria Version 4.0 [23] at each follow-up.

Overall survival (OS) in both groups was defined as the time period from the start of TKIs plus ICls treatment to the patient's death or survival to the cut-off date (January 31, 2021). PFS was defined as either radiologic evidence of tumor progression at the time of TKIs plus ICls therapy initiation or patient death/ survival until the cutoff date, whichever occurred first.

\section{Statistical Analyses}

The population baseline characteristics were determined using descriptive statistical methods, with categorical variables represented by median and quaternary intervals while continuous variables represented by median and $95 \% \mathrm{Cl}$. Categorical variables were tested by $\chi^{2}$ test or Fisher's exact test, and continuous variables were tested by $t$ test or $u$ test. Kaplan-Meier survival analysis was used for PFS and OS, while log-rank was used to test for differences between groups. Cox proportional hazard regression models were utilized for univariate and multivariate analyses of OS in the total population of patients. The dichotomous variables $p \leq 0.1$ from univariate analyses were included in the multivariate analysis to describe the prognostic correlation of the potential survival predictors. SPSS Statistics (version 26.0; IBM, Armonk, NY) was employed for all statistical analyses, and bilateral tests were used for all statistical tests. A two-sided $p$ value less than 0.05 indicated statistical significance.

\section{Results}


147 advanced HCC patients who received TKIs plus ICls with or without TACE between July 2017 and April 2020 were evaluated in this study. Among them, 98 patients who underwent TACE during TKIs plus ICls treatment were included in the TACE group (median age, 52 years; interquartile range [IQR] 42-62 years; 87 men), while the other 49 patients were included in the non-TACE group (median age, 53 years; IQR 47-63 years; 47 men). Baseline characteristics of the two groups are shown in Table 1, including age, gender, hepatitis B surface antigen, tumor size, tumor number, tumor involvement, main portal vein tumor thrombus, macrovascular invasion, extrahepatic metastasis, ascites, albumin (ALB), total bilirubin (TBIL), platelet count (PLT), prothrombin time (PT), a-fetoprotein (AFP) level, Child-Pugh Score, BCLC stage and history of locoregional therapy. There was no significant difference between the two groups of

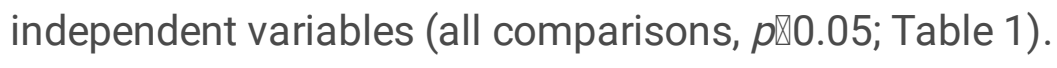


Table 1

Baseline patient characteristics

\begin{tabular}{|c|c|c|c|}
\hline Variable & $\begin{array}{l}\text { TACE group } \\
(n=98)\end{array}$ & $\begin{array}{l}\text { Non-TACE group } \\
(n=49)\end{array}$ & $p$ value \\
\hline Mean age (y) & $52(42-62)$ & $53(47-63)$ & 0.108 \\
\hline Sex & & & 0.150 \\
\hline Male & $87(88.8)$ & 47 (95.9) & \\
\hline Female & $11(11.2)$ & $2(4.1)$ & \\
\hline hepatitis B surface antigen & & & 0.862 \\
\hline Positive & $85(86.7)$ & $43(87.8)$ & \\
\hline Negative & $13(13.3)$ & $6(12.2)$ & \\
\hline Maximum diameter of intrahepatic tumors $(\mathrm{cm})$ & $8.8(6.4-12.4)$ & $7.8(3.8-12.6)$ & 0.209 \\
\hline No. of intrahepatic tumors & & & 0.692 \\
\hline$\leq 3$ & $27(27.6)$ & $12(24.5)$ & \\
\hline$>3$ & $71(72.4)$ & $37(75.5)$ & \\
\hline Tumor involvement & & & 0.806 \\
\hline Unilobar & $34(34.7)$ & $16(32.7)$ & \\
\hline Bilobar & $64(65.3)$ & $33(67.3)$ & \\
\hline Main portal vein tumor thrombus & & & 0.744 \\
\hline Yes & $14(14.3)$ & $8(16.3)$ & \\
\hline No & $84(85.7)$ & $41(83.7)$ & \\
\hline Macrovascular invasion 9 & & & 0.098 \\
\hline Yes & $73(74.5)$ & $30(61.2)$ & \\
\hline No & $25(25.5)$ & $19(38.8)$ & \\
\hline Extrahepatic metastasis & & & 0.726 \\
\hline Yes & $49(50.0)$ & $26(53.1)$ & \\
\hline No & $49(50.0)$ & $23(46.9)$ & \\
\hline Ascites & & & 0.468 \\
\hline Yes & $34(34.7)$ & $20(40.8)$ & \\
\hline No & 64 (65.3) & $29(59.2)$ & \\
\hline
\end{tabular}




\begin{tabular}{|llll|}
\hline Variable & $\begin{array}{l}\text { TACE group } \\
(\boldsymbol{n}=\mathbf{9 8})\end{array}$ & $\begin{array}{l}\text { Non-TACE group } \\
(\boldsymbol{n}=\mathbf{4 9})\end{array}$ & $\boldsymbol{p}$ value \\
\hline Median ALB $(\mathrm{g} / \mathrm{L})$ & $35.8(33.4-38.8)$ & $35.5(31.3-40.2)$ & 0.887 \\
\hline Median total bilirubin $(\mathrm{mmol} / \mathrm{L})$ & $16.7(11.6-27.0)$ & $11.5(9.2-19.9)$ & 0.148 \\
\hline Median platelet count $\left(\times 10^{\wedge} 9 / \mathrm{L}\right)$ & $158(107-232)$ & $114(91-224)$ & 0.767 \\
\hline Median PT (second) & $14.2(13.4-15.1)$ & $14.0(13.3-14.7)$ & 0.617 \\
\hline
\end{tabular}

Table 1

(continued) Baseline Patient Characteristics

\begin{tabular}{|c|c|c|c|}
\hline Variable & TACE Group $(n=98)$ & Non-TACE Group $(n=49)$ & $P$ value \\
\hline AFP (ng/mL) & & & 0.554 \\
\hline$\nabla 200$ & $59(60.2)$ & $27(55.1)$ & \\
\hline$\leq 200$ & $39(39.8)$ & $22(44.9)$ & \\
\hline Child-Pugh liver function class & & & 0.234 \\
\hline A5-A6 & $75(76.5)$ & $33(67.3)$ & \\
\hline B7-B9 & $23(23.5)$ & $16(32.7)$ & \\
\hline BCLC stage & & & 0.728 \\
\hline B & $12(12.2)$ & $7(14.3)$ & \\
\hline C & $86(87.8)$ & $42(85.7)$ & \\
\hline History of locoregional therapy & $69(70.4)$ & $40(81.6)$ & 0.143 \\
\hline \multicolumn{4}{|c|}{$\begin{array}{l}\text { Except where indicated, data are numbers of patients, with percentages in parentheses. Abbreviations: } \\
\text { AFP, a-fetoprotein; ALB, albumin; BCLC, Barcelona Clinic Liver Cancer; PT, Prothrombin time; TACE, } \\
\text { transarterial chemoembolization. Non-TACE group refers to patients who who received systemic } \\
\text { combination therapy without transarterial chemoembolization. }\end{array}$} \\
\hline \multicolumn{4}{|c|}{ * Numbers in parentheses are the interquartile range. } \\
\hline $\begin{array}{l}\text { Some patients had hepatic ve } \\
\text { one in the non-TACE group). }\end{array}$ & invasion and no port & ein invasion (five in the TAC & oup and \\
\hline
\end{tabular}

By the cut-off date (January 31, 2021), 45 (45.5\%) of the 98 patients in the TACE group and 31 (63.3\%) of the 49 patients in the non-TACE group died. 7 (7.1\%) patients in the TACE group and 4 (8.2\%) patients in the non-TACE group continued TKIs plus ICls therapy until the cut-off date, respectively. Sequential treatment of patients in the two groups after TKIs plus ICls treatment was presented in Table 2. 
Table 2

Sequential treatment after TKIs plus ICls therapy

\begin{tabular}{|lll|}
\hline Variable & $\begin{array}{l}\text { TACE group } \\
(\boldsymbol{n}=\mathbf{4 6})\end{array}$ & $\begin{array}{l}\text { Non-TACE group } \\
(\boldsymbol{n}=\mathbf{1 4})\end{array}$ \\
\hline Downstaging hepatectomy & $1(2.2)$ & $1(7.1)$ \\
\hline Downstaging ablation & $2(4.3)$ & $1(7.1)$ \\
\hline TACE & $19(41.3)$ & $4(28.6)$ \\
\hline HAIC & $4(8.7)$ & $3(21.4)$ \\
\hline Radiotherapy & $4(8.7)$ & $1(7.1)$ \\
\hline Replace TKIs & $23(50.0)$ & $9(64.3)$ \\
\hline Best supportive care & $5(10.9)$ & $1(7.1)$ \\
\hline $\begin{array}{l}\text { Except where indicated, data are numbers of patients, with percentages in parentheses. Abbreviations: } \\
\text { ICls, Immune checkpoint inhibitors; HAIC, hepatic artery infusion chemotherapy; TACE, transarterial } \\
\text { chemoembolization; TKIs, tyrosine kinase inhibitors. }\end{array}$ \\
\hline
\end{tabular}

\section{Treatment Outcomes and Survival Analysis in the Overall Cohort}

The median OS of the TACE group and the non-TACE group were 19.3months (95\% $\mathrm{Cl} 14.6-24.1)$ and 10.8 months $(95 \% \mathrm{Cl} 6.7-14.9)$, respectively, meaning that the TACE group had significantly longer $(p=$ 0.010) OS time (Fig. 2a). Median PFS for the TACE group was 9.3 months $(95 \% \mathrm{Cl} 7.5-11.1)$, and for the non-TACE group, it was 7.6 months $(95 \% \mathrm{Cl} 5.8-9.4)(p=0.308)$ (Fig. 2b).

As shown in Table 3, 17 clinical factors were included in the Cox proportional hazard regression models. The multivariate analysis indicated that the TACE group still had a significant advantage regarding patients' survival over non-TACE group ( $p=0.004$, hazard ratio $[\mathrm{HR}]=2.00,95 \% \mathrm{Cl} 1.25-3.19)$. In addition, the main portal vein tumor thrombus ( $p=0.001, \mathrm{HR}=0.37,95 \% \mathrm{Cl} 0.20-0.66)$, and the AFP $>200 \mathrm{ng} / \mathrm{mL}$ $(p=0.001, \mathrm{HR}=0.41,95 \% \mathrm{Cl} 0.27-0.71)$ were also independent predictors associated with OS (Fig. 3 ). 
Table 3

Risk factors analysis for death

Univariable Analysis

Hazard $\quad 95 \% \mathrm{Cl}$

Ratio

TACE group

Age $>60 y$

0.55

0.73

0.74

1.21

Positive for HBsAg

Number of intrahepatic

tumors $>3$

Involvement of bilobar

Main portal vein tumor

thrombus

Macrovascular invasion

Extrahepatic metastasis

1.30

1.18

1.85

2.00

$0.35-$

0.87

$0.43-$

1.23

$0.35-$

1.54

$0.60-$

2.44

$0.97-$

2.41

2.08

$1.16-$

3.72

$1.10-$

3.12

1.14-

3.48

$0.71-$

1.95

0.82
Size of main tumor $>10 \mathrm{~cm}$

2.05

Ascites

ALB $\leq 36 \mathrm{~g} / \mathrm{L}$

1.08

1.22

0.77-

1.94

0.69-

1.71

total bilirubin $>34 \mathrm{mmol} / \mathrm{L}$

1.10

0.55

2.22

Platelet count $\leq$

$100 \times 10^{\wedge} 9 / \mathrm{L}$

AFP $>200 n g / m L$

2.02

0.76

$0.43-$

1.34

$1.23-$

3.32

1.21

1.99
$0.72-$

2.02
Child-Pugh class B

$0.86-$

BCLC stage $\mathrm{C}$
Multivariable Analysis

Hazard
Ratio $\quad 95 \% \mathrm{Cl} \quad \stackrel{p}{\text { Value }}$

$0.011 \quad 0.50$

$0.31-$

0.80

0.004

0.233

0.428

0.586

0.070

0.99

$0.60-$

0.952

1.62

$0.89-$

0.113

3.14

0.92-

2.72

0.103

1.58

$1.52-$ 4.90

0.001

2.73

0.525

0.267

0.400

0.730

0.781

0.340

4.58

0.006

2.42

$1.41-$

0.001

4.13

0.474

0.107 
All 147 patients were analyzed. $p \leq 0.05$ was considered to indicate statistical significance. Abbreviations: AFP, a-fetoprotein; ALB, albumin; BCLC, Barcelona Clinic Liver Cancer; PT, Prothrombin time.

Details of the different combinations of TKIs and ICls are shown in Table 4. The radiological evaluation outcomes of two groups of patients with advanced HCC are revealed in Table 5. According to the RECIST 1.1 , the ORR of the TACE group was $37.8 \%$ (37/98), and this value of the non-TACE group was $26.5 \%$ $(13 / 49)(p=0.176)$, while the DCR was $90.1 \%(89 / 98)$ for the TACE group and $63.3 \%(31 / 49)$ for the nonTACE group $(p=0.002)$, respectively. According to MRECIST, the ORR and DCR of the TACE group were significantly higher than that of the non-TACE group (ORR $74.5 \%$ vs. $40.8 \%, P \otimes 0.001$; DCR $83.7 \%$ vs. $67.3 \%, p=0.012)$. A representative case receiving systemic combination therapy with TACE is shown in Fig. 4.

Table 4

Collocation selection of systemic combination therapy at baseline

\begin{tabular}{|lll|}
\hline TACE group, non-TACE group & Sorafenib & Lenvatinib \\
\hline Nivolumab & $14(14.3), 5(10.2)$ & $7(7.1), 1(2.0)$ \\
\hline Pembrolizumab & $4(4.1), 2(4.1)$ & $14(14.3), 4(8.2)$ \\
\hline Camrelizumab & $40(40.8), 30(61.2)$ & $19(19.4), 7(14.3)$ \\
\hline Except where indicated, data are numbers of patients, with percentages in parentheses. \\
\hline
\end{tabular}


Table 5

Outcomes in patients with advanced HCC treated with TACE group and non-TACE group

\begin{tabular}{|c|c|c|c|c|c|c|}
\hline & \multicolumn{3}{|l|}{ RECIST 1.1} & \multicolumn{3}{|l|}{ mRECIST } \\
\hline Variable & $\begin{array}{l}\text { TACE group } \\
(\mathrm{n}=98)\end{array}$ & $\begin{array}{l}\text { Non-TACE } \\
\text { group }(n=49)\end{array}$ & $p_{\text {Value }}$ & $\begin{array}{l}\text { TACE } \\
\text { group ( } \mathrm{n}= \\
\text { 98) }\end{array}$ & $\begin{array}{l}\text { Non-TACE } \\
\text { group }(n=49)\end{array}$ & $p_{\text {Value }}$ \\
\hline \multicolumn{7}{|l|}{$\begin{array}{l}\text { Best } \\
\text { response }\end{array}$} \\
\hline CR & $0(0)$ & $0(0)$ & & $20(20.4)$ & $4(8.2)$ & \\
\hline PR & 37 (37.8) & $13(26.5)$ & & $53(54.1)$ & $16(32.7)$ & \\
\hline SD & $52(53.1)$ & $22(44.9)$ & & $16(16.3)$ & $17(34.7)$ & \\
\hline PD & $9(9.2)$ & $14(28.6)$ & & $9(9.2)$ & $12(24.5)$ & \\
\hline $\begin{array}{l}\text { ORR (CR + } \\
\text { PR) }\end{array}$ & 37 (37.8) & $13(26.5)$ & 0.176 & $73(74.5)$ & $20(40.8)$ & $\otimes 0.001$ \\
\hline $\begin{array}{l}\mathrm{DCR}(\mathrm{CR}+ \\
\mathrm{PR}+\mathrm{SD})\end{array}$ & $89(90.1)$ & 31 (63.3) & 0.002 & 82 (83.7) & 33 (67.3) & 0.012 \\
\hline \multicolumn{7}{|c|}{$\begin{array}{l}\text { Unless otherwise indicated, data are numbers of patients and data in parentheses are percentages. } \\
\text { Outcomes were determined according the Response Evaluation Criteria in Solid Tumors, version } 1.1 \\
\text { and modified Response Evaluation Criteria in Solid Tumors. Non-TACE group refers to patients who } \\
\text { who received systemic combination therapy without transarterial chemoembolization. Abbreviations: } \\
\text { CR, complete response; DCR, disease control rate; ORR, objective response rate; PD, progression } \\
\text { disease; PR, partial response; SD, stable disease. }\end{array}$} \\
\hline
\end{tabular}

\section{Complications}

Table 6 reveals an incidence that greater than 10\% regarding the TACE-related and drug-related AEs for all grades, and grades 3-4 in the TACE group and the non-TACE group. The median treatment duration of TKIs plus ICls for the TACE group was 5.6 months $(95 \% \mathrm{Cl} 3.0-11.2)$, and it was 3.4 months $(95 \% \mathrm{Cl} 0.9-$ 5.8) for the non-TACE group. Patients in the TACE group received a total of 224 TACE procedures, with a median of 3.0 (IQR 1.0-3.0) times per patient. 
Table 6

Adverse events with an incidence of more than $10 \%$ in either group

\begin{tabular}{|c|c|c|c|c|}
\hline & $\begin{array}{l}\text { TACE group } \\
(\mathrm{n}=98)\end{array}$ & & $\begin{array}{l}\text { Non-TACE } \\
(n=49)\end{array}$ & \\
\hline Parameter & Any Grade & Grade 3-4 & Any Grade & Grade 3-4 \\
\hline Abdominal pain & $73(74.5)$ & $10(10.2)$ & $21(42.9)$ & $0(0)$ \\
\hline AST/ALT increased & $55(56.1)$ & $4(4.1)$ & $12(24.5)$ & $1(2.0)$ \\
\hline Constipation & 35 (35.7) & $0(0)$ & $13(26.5)$ & $0(0)$ \\
\hline Pyrexia & $34(34.7)$ & $7(7.1)$ & $14(28.6)$ & $2(4.1)$ \\
\hline Hypertension & 31 (31.6) & $9(9.2)$ & $13(26.6)$ & $4(8.2)$ \\
\hline PLT decreased & $26(26.5)$ & $3(3.1)$ & $20(40.9)$ & $2(4.1)$ \\
\hline Fatigue & $24(24.5)$ & $4(4.1)$ & $15(26.5)$ & $1(2.0)$ \\
\hline Weight decreased & $24(24.5)$ & $0(0)$ & $13(26.5)$ & $0(0)$ \\
\hline Diarrhea & $22(22.4)$ & $0(0)$ & $7(14.4)$ & $0(0)$ \\
\hline Rash & $21(21.4)$ & $1(1.0)$ & $10(20.4)$ & $1(2.0)$ \\
\hline Pneumonitis & 13 (13.3) & $2(2.0)$ & $5(10.2)$ & $2(4.1)$ \\
\hline Nausea & $11(11.2)$ & $2(2.0)$ & $6(12.2)$ & $0(0)$ \\
\hline Proteinuria & $11(11.2)$ & $0(0)$ & $6(12.2)$ & $1(2.0)$ \\
\hline TBIL increased & $10(10.2)$ & $2(2.0)$ & 8 (16.3) & $2(4.1)$ \\
\hline
\end{tabular}

The incidence of grade $3-4$ toxicities in the overall population was $32.0 \%(47 / 147) .98 .0 \%(96 / 98)$ and $33.7 \%(33 / 98)$ of patients in the TACE group and $98.0 \%$ (48/49) and $28.6 \%(14 / 49)$ of patients in the nonTACE group experienced any grade of AEs and grade 3-4 AEs, respectively. No patients discontinued TKIs plus ICls treatment due to TACE-related toxic side effects. There were no previously unreported AEs or death because of TACE or TKIs plus ICls therapy. 35 (35.7\%) patients from the TACE group and 16 (32.7\%) patients from the non-TACE group were prescribed dose change or interruption due to treatmentrelated toxicity of systemic combination therapy, respectively. Grade 3-4 abdominal pain was observed more frequently in the TACE group compared to the non-TACE group ( $10.1 \% \mathrm{vs.} 0 \%)$, but symptoms were effectively controlled with analgesic medication. The overall incidence of grade 3-4 AEs in TACE group was similar to that in non-TACE group (33.7\% vs. $28.6 \%, p=0.532)$ (Table 7$)$. 
Table 7

Comparison of adverse events between TACE group and non-TACE group

\begin{tabular}{|lllll|}
\hline Parameter & $\begin{array}{l}\text { TACE group } \\
(\mathbf{n = 9 8 )}\end{array}$ & $\begin{array}{l}\text { Non-TACE group } \\
(\mathbf{n = 4 9 )}\end{array}$ & $\begin{array}{l}\text { All patients } \\
(\mathbf{n}=\mathbf{1 4 7 )}\end{array}$ & p value \\
\hline Any Grade & $96(98.0)$ & $48(98.0)$ & $144(98.0)$ & 1.0 \\
\hline Grade 3-4 & $33(33.7)$ & $14(28.6)$ & $47(32.0)$ & 0.532 \\
\hline \multicolumn{4}{l}{$\begin{array}{l}\text { Data in parentheses are percentages. Non-TACE group refers to patients who who received systemic } \\
\text { combination therapy without transarterial chemoembolization. }\end{array}$} \\
\hline
\end{tabular}

\section{Discussion}

In previous studies regarding the treatment of advanced HCC, the clinical effectiveness of TACE in the therapeutic combination of TKIs and ICls has not been explored. In this study, we firstly validated the positive effect of TACE in HCC patients treated with TKIs and ICls. We described that the OS of TKIs plus ICls with TACE was significantly longer than systemic combination therapy without TACE (19.3 months vs. 10.8 months, $p=0.010$ ), which is consistent with the analysis using the cox proportional hazard regression models. The PFS of TACE combined with TKIs and ICls was also improved compared with that of treatment without TACE (9.3 months vs. 7.6 months, $p=0.308)$, although the statistical difference was not significant. According to the mRECIST, the objective response rate (ORR) of the TACE group was higher than the non-TACE group as well (ORR $74.5 \%$ vs. $40.8 \%, p \otimes 0.001$ ). These results provide hypothesis-generating data for the design of prospective, randomized, phase 2 efficacy trials.

Several reasons may explain the improvement of the survival time for the advanced HCC patients brought by the addition of TACE into the treatment of TKIs plus ICls. On the one hand, despite the fact that TACE has long been recognized to promise prolonged survival in HCC patients, the TACE-induced tumor hypoxia and up-regulation of vascular endothelial growth factor (VEGF) and platelet- derived growth factor (PDGF) can also be a cause of tumor recurrence [24,25]. What's more, enhanced VEGF expression promotes immune evasion, because of which some HCC patients fail to benefit from immunotherapy. TKIs can inhibit TACE-related tumor recurrence by blocking VEGF receptors and preventing the surge of pro-angiogenic factors, and they can reprogram the immunosuppressive microenvironment around tumors into an immune-stimulating environment, in which conditions the efficacy of immunotherapy is enhanced $[26,27]$. On the other hand, TACE induces cell death to release tumor antigens, thus improving the immunotherapy efficacy [28]. TACE, especially those induced by locally infused chemotherapeutic agents (e.g., doxorubicin, a classic chemotherapeutic agent that induces immunogenic cell death), causes changes in tumor-specific and innate immune responses, thus inducing anti-tumor immunity, and thereby enhances the ICls response [29]. Potentially positive interactions among these treatment modalities have further improved survival outcomes in patients with advanced HCC. However, the exact molecular mechanism still needs to be discovered. 
Compared to the OS revealed by phase Ib study of lenvatinib plus pembrolizumab in patients with unresectable HCC [30], the survival outcome in TKIs plus ICls with TACE in our study did not appear to be further improved. The underlying reason could be the worse baseline status in this study, the inclusion of more than $70 \%$ of patients with macrovascular invasion and nearly one-third of patients with Child-Pugh class $B$ liver disease. Exceptionally, the median maximum intrahepatic tumor diameter in these patients was approximately $8 \mathrm{~cm}$, and this higher tumor burden also affected the survival improvement. All of the above-described reasons are vital factors that limit improvement. As a real-world cohort study, the baseline status of the enrolled population was worse than those in previous clinical studies, making this study more representative of the actual clinical treatment effect of TKIs plus ICls with TACE.

In this study, the treatment of TACE combined TKIs and ICls was validated to be clinically effective and safe. The classification, incidence and severity of AEs in the whole population were also divided into two groups according to whether TACE was done or not during systemic combination therapy for comparative analysis. We found that the difference in AEs between the TACE group and non-TACE group was mainly reflected in post-embolism syndromes after TACE. Especially, the incidence of abdominal pain in the TACE group is higher than that of TKIs plus ICls without TACE, regardless of disease level or grade. All patients with grade $3-4$ abdominal pain occurred within 3 days after TACE, which can be effectively controlled by analgesic medication. In addition, all types of AEs were similar in both groups and were generally consistent with safety conclusions from other known trials, and TACE did not lead to more severe AEs associated with systemic combination therapy.

There are several limitations in this study. First, as a retrospective study with a real-world cohort, there is an inevitable selection bias affecting the results. Second, numerous combinations of TKIs and ICls were involved in this study, while it is impossible to determine the best combination due to the small sample size. Thirdly, the diagnosis of HCC in most patients was determined by combining contrast-enhanced CT or MR imaging findings and a-fetoprotein level with no pathological confirmation. Research and analysis at the cellular and molecular levels also cannot be carried out due to the lack of tumor tissue specimens before and after treatment in these patients. Finally, although the baseline status after grouping was balanced, the limited number of enrolled patients in the study affected the validity of the analysis.

In summary, TACE combined with TKIs and ICls had a better OS than TKIs plus ICls without TACE for the treatment of advanced HCC. What's more, TACE did not lead to more severe AEs associated with TKIs plus ICls therapy. However, more extensive prospective clinical trials are needed to further confirm these findings.

\section{Declarations}

Conflicts of Interest Yue Hu, Tao Pan, Xi Cai, Quansheng He, Bing Hu, Yubao Zheng, Ting Jiang, Mingsheng Huang, Zaibo Jiang, JunWei Chen and Chun Wu declare that they have no conflict of interest.

Ethics approval The study protocol was approved by the ethical committee of the Third Affiliated Hospital of Sun Yat-Sen University. All procedures followed were in accordance with the ethical standards of the 
responsible committee on human experimentation (institutional and national) and with the Helsinki Declaration of 1975, as revised in 2008 (5).

Informed consent Patient consent was waived due to this was a retrospective study.

Code availability: Not applicable.

\section{References}

1. European Association for the Study of the Liver. Electronic address eee, European Association for the Study of the L. EASL Clinical Practice Guidelines: Management of hepatocellular carcinoma. J Hepatol. 2018;69(1):182-236

2. Morise Z, Kawabe N, Tomishige H, Nagata H, Kawase J, Arakawa S, et al. Recent advances in the surgical treatment of hepatocellular carcinoma. World J Gastroenterol. 2014;20(39):14381-14392

3. Llovet JM, Ricci S, Mazzaferro V, Hilgard P, Gane E, Blanc J, et al. Sorafenib in Advanced Hepatocellular Carcinoma. New England Journal of Medicine. 2008;359(4):378-390

4. Kudo M, Finn RS, Qin S, Han KH, Ikeda K, Piscaglia F, et al. Lenvatinib versus sorafenib in first-line treatment of patients with unresectable hepatocellular carcinoma: a randomised phase 3 non-inferiority trial. The Lancet. 2018;391(10126):1163-1173

5. Chen LT, Martinelli E, Cheng AL, Pentheroudakis G, Qin S, Bhattacharyya GS, et al. Pan-Asian adapted ESMO Clinical Practice Guidelines for the management of patients with intermediate and advanced/relapsed hepatocellular carcinoma: a TOS-ESMO initiative endorsed by CSCO, ISMPO, JSMO, KSMO, MOS and SSO. Ann Oncol. 2020;31(3):334-351

6. Heimbach JK, Kulik LM, Finn RS, Sirlin CB, Abecassis MM, Roberts LR, et al. AASLD guidelines for the treatment of hepatocellular carcinoma. Hepatology. 2018;67(1):358-380

7. Xie DY, Ren ZG, Zhou J, Fan J, Gao Q. 2019 Chinese clinical guidelines for the management of hepatocellular carcinoma: updates and insights. Hepatobiliary Surg Nutr. 2020;9(4):452-463

8. El-Khoueiry AB, Sangro B, Yau T, Crocenzi TS, Kudo M, Hsu C, et al. Nivolumab in patients with advanced hepatocellular carcinoma (CheckMate 040): an open-label, non-comparative, phase 1/2 dose escalation and expansion trial. The Lancet. 2017;389(10088):2492-2502

9. Finn RS, Ryoo BY, Merle P, Kudo M, Bouattour M, Lim HY, et al. Pembrolizumab as second-line therapy in patients with advanced hepatocellular carcinoma in KEYNOTE-240: a randomized, doubleblind, phase III trial. J Clin Oncol. 2020; 38(3):193-202

10. Qin S, Ren Z, Meng Z, Chen Z, Chai X, Xiong J, et al. Camrelizumab in patients with previously treated advanced hepatocellular carcinoma: a multicentre, open-label, parallel-group, randomised, phase 
2 trial. The Lancet Oncology. 2020;21(4):571-580

11. Finn RS, Chan SL, Zhu AX, Knox J, Cheng AL, Siegel AB, et al. KEYNOTE-240: Phase 3, randomized study of pembrolizumab (pembro) vs best supportive care (BSC) for second-line advanced hepatocellular carcinoma (HCC). Annals of Oncology. 2017;28(Supple_5):v266

12. Fizazi K, Maillard A, Penel N, Baciarello G, Allouache D, Daugaard G, et al. A phase III trial of empiric chemotherapy with cisplatin and gemcitabine or systemic treatment tailored by molecular gene expression analysis in patients with carcinomas of an unknown primary (CUP) site (GEFCAPI 04). Annals of Oncology. 2019;30(Supple_5):v851-v934

13. Hegde PS, Wallin JJ, Mancao C. Predictive markers of anti-VEGF and emerging role of angiogenesis inhibitors as immunotherapeutics. Semin Cancer Biol. 2018;52:117-124

14. Llovet J, Shepard KV, Finn RS, Ikeda M, Sung M, Baron AD, et al. A phase $1 b$ trial of lenvatinib plus pembrolizumab in unresectable hepatocellular carcinoma: Upated results. Ann Oncol. 2019; 30(Supple_5): v253-v324

15. Finn RS, Qin S, Ikeda M, Galle PR, Ducreux M, Kim TY, et al. Atezolizumab plus Bevacizumab in Unresectable Hepatocellular Carcinoma. N Engl J Med. 2020;382(20):1894-1905

16. Forner A, Reig M, Bruix J. Hepatocellular carcinoma. The Lancet. 2018;391(10127):1301-1314

17. Pinter M, Peck-Radosavljevic M. Review article: systemic treatment of hepatocellular carcinoma. Aliment Pharmacol Ther. 2018;48(6):598-609.

18. Kudo M, Ueshima K, Ikeda M, Torimura T, Tanabe N, Aikata H, et al. Randomised, multicentre prospective trial of transarterial chemoembolisation (TACE) plus sorafenib as compared with TACE alone in patients with hepatocellular carcinoma: TACTICS trial. Gut. 2020;69(8):1492-1501

19. Greten TF, Mauda-Havakuk M, Heinrich B, Korangy F, Wood BJ. Combined locoregionalimmunotherapy for liver cancer. J Hepatol. 2019;70(5):999-1007

20. Zhu K, Huang J, Lai L, Huang W, Cai M, Zhou J, et al. Medium or Large Hepatocellular Carcinoma: Sorafenib Combined with Transarterial Chemoembolization and Radiofrequency Ablation. Radiology. 2018;288(1):300-307

21. Eisenhauer EA, Therasse P, Bogaerts J, Schwartz LH, Sargent D, Ford R, et al. New response evaluation criteria in solid tumours: revised RECIST guideline (version 1.1) Eur J Cancer. 2009;45(2):228247

22. Lencioni R, Llovet JM. Modified RECIST (mRECIST) assessment for hepatocellular carcinoma. Semin Liver Dis. 2010;30(1):52-60 
23. National Cancer Institute. Protocol development. Cancer therapy evaluation program.

https://ctep.cancer.gov/protocolDevelopment/ electronic_applications/ctc.htm (accessed on February 10, 2020).

24. Wang B, Xu H, Gao ZQ, Ning HF, Sun YQ, Cao GW. Increased expression of vascular endothelial growth factor in hepatocellular carcinoma after transcatheter arterial chemoembolization. Acta Radiol. 2008;49(5):523-529

25. Piret JP, Cosse JP, Ninane N, Raes M, Michiels C. Hypoxia protects HepG2 cells against etoposideinduced apoptosis via a HIF-1-independent pathway. Exp Cell Res. 2006;312(15):2908-2920

26. Jiang H, Meng Q, Tan H, Pan S, Sun B, Xu R, et al. Antiangiogenic therapy enhances the efficacy of transcatheter arterial embolization for hepatocellular carcinomas. Int J Cancer. 2007;121(2):416-424

27. Kudo M. Scientific Rationale for Combined Immunotherapy with PD-1/PD-L1 Antibodies and VEGF Inhibitors in Advanced Hepatocellular Carcinoma. Cancers (Basel). 2020;12(5):1089

28. Ayaru L, Pereira SP, Alisa A, Pathan AA, Williams R, Davidson B, et al. Unmasking of -fetoproteinspecific CD4+ T cell responses in hepatocellular carcinoma patients undergoing embolization. $J$ Immunol. 2007;178(3):1914-1922

29. Heinrich S, Castven D, Galle PR, Marquardt JU. Translational Considerations to Improve Response and Overcome Therapy Resistance in Immunotherapy for Hepatocellular Carcinoma. Cancers (Basel). 2020;12(9):2495

30. Finn RS, Ikeda M, Zhu AX, Sung MW, Baron AD, Kudo M, et al. Phase lb Study of Lenvatinib Plus Pembrolizumab in Patients With Unresectable Hepatocellular Carcinoma. J Clin Oncol. 2020;38(26):29602970

\section{Figures}




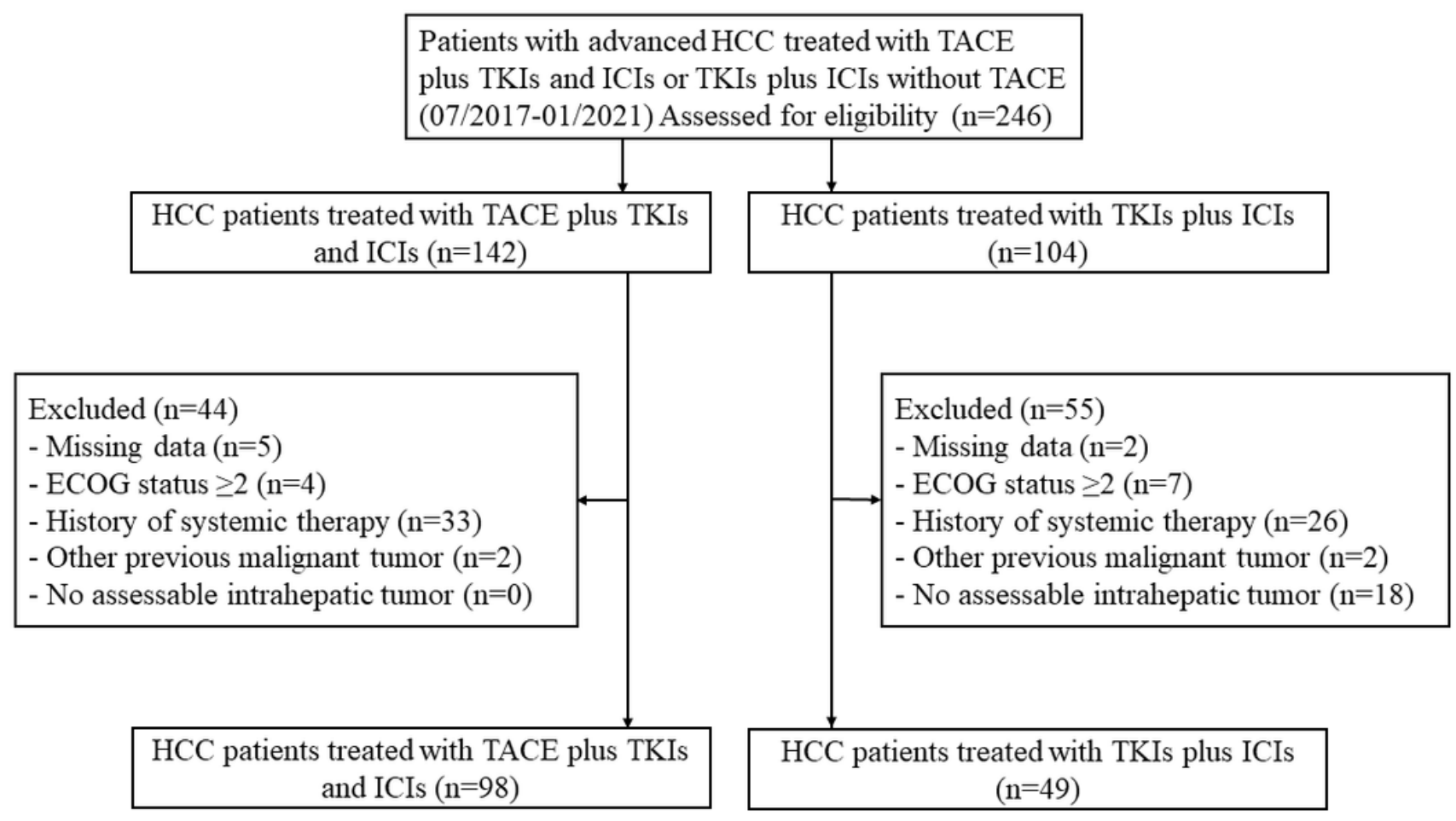

\section{Figure 1}

Flowchart of patient inclusion and outcomes. Abbreviations: ICls, immune checkpoint inhibitors; ECOG, Eastern Cooperative Oncology Group; HCC, hepatocellular carcinoma; TACE, transarterial chemoembolization; TKIs, tyrosine kinase inhibitors 

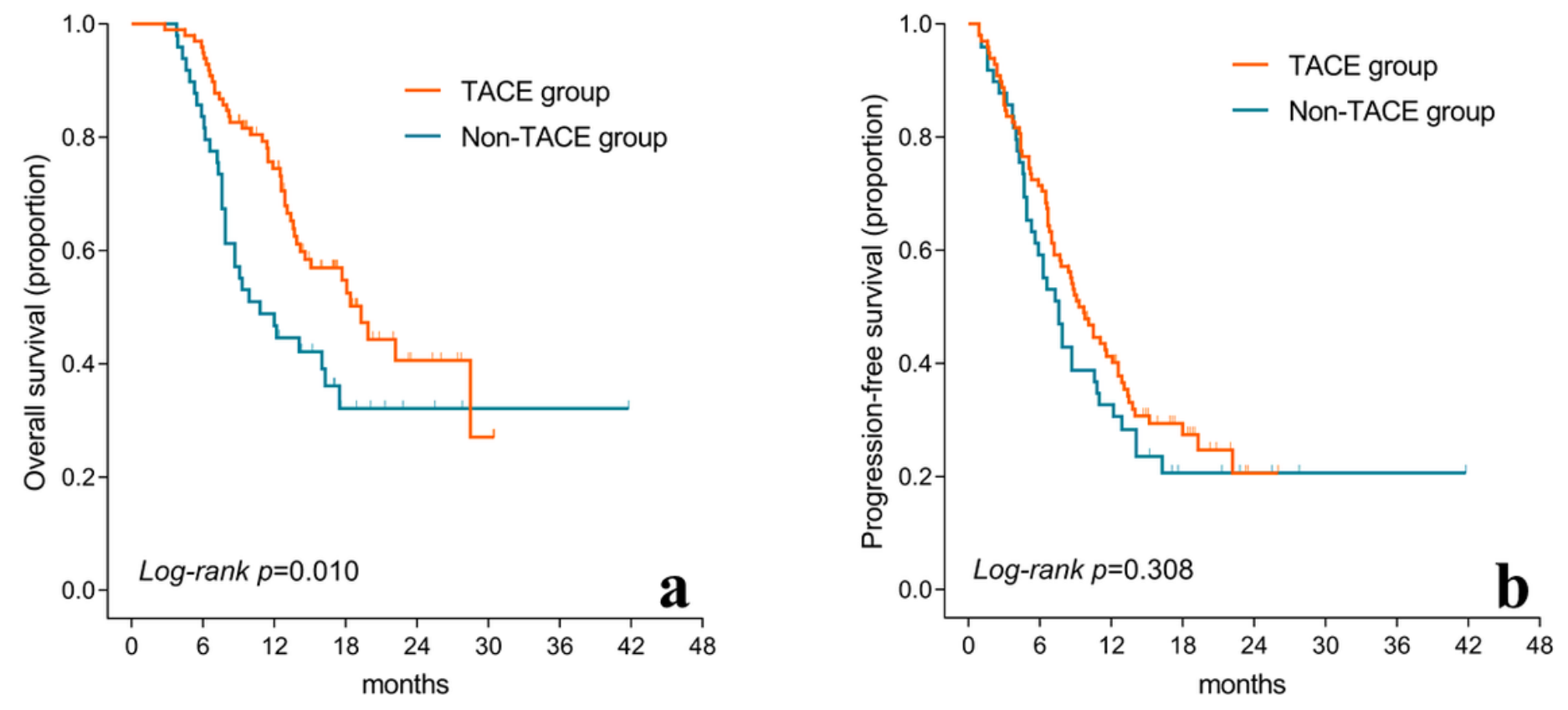

\section{Figure 2}

Kaplan-Meier curves of survival outcomes in patients with advanced hepatocellular carcinoma who received tyrosine kinase inhibitors plus immune checkpoint inhibitors with or without transarterial chemoembolization (TACE). (a) cumulative overall survival (OS) curves. (b) cumulative progression-free survival (PFS) curves 

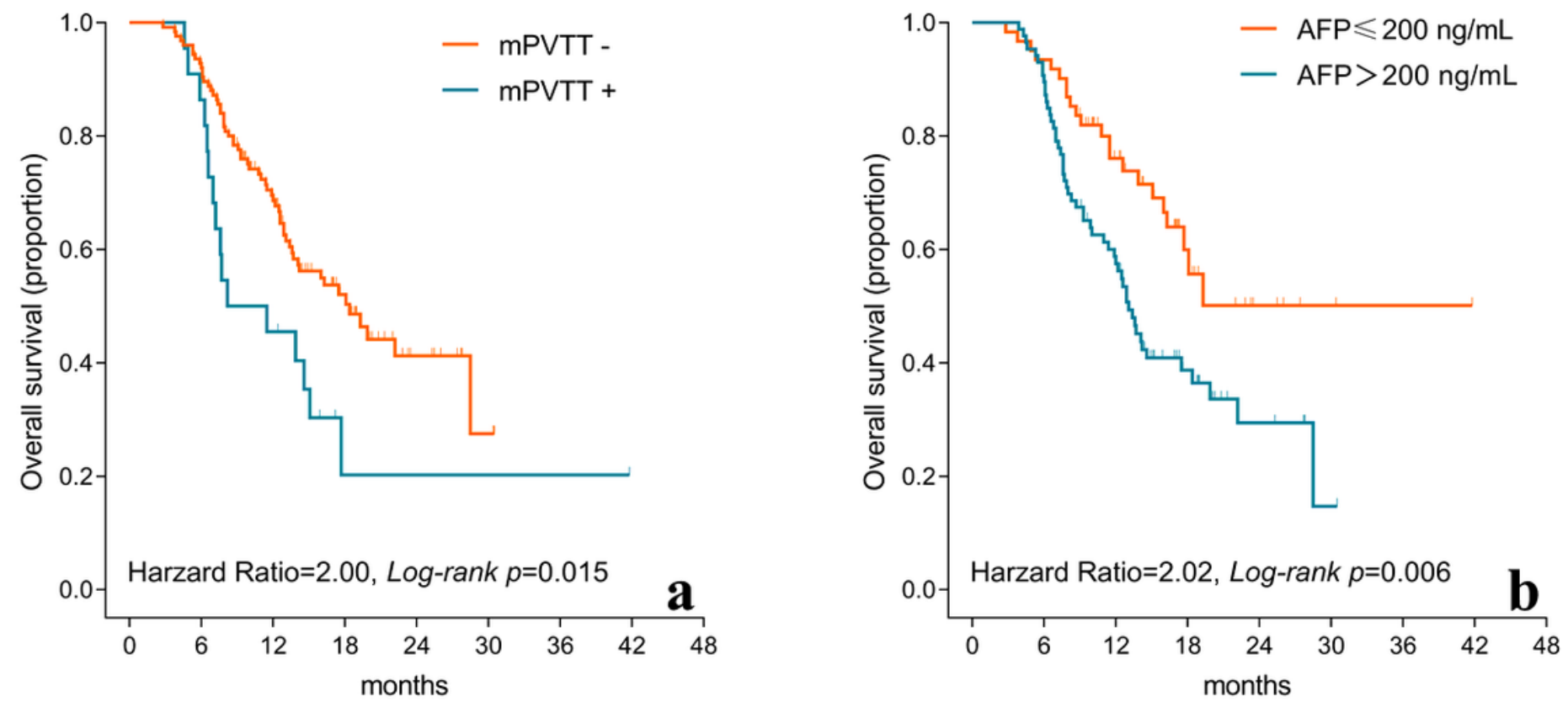

\section{Figure 3}

Curves nerated with univariate analysis (the log-rank test) to determine prognostic factors for overall survival (OS) in all patients. (a) Cumulative OS curve according to the presence or absence of main portal vein tumor thrombus (mPVTT). (b) Cumulative OS curve according to a-fetoprotein (AFP) levels ( $\leq 200$ $\mathrm{ng} / \mathrm{mL} v \mathrm{v} \bowtie 200 \mathrm{ng} / \mathrm{mL})$ 


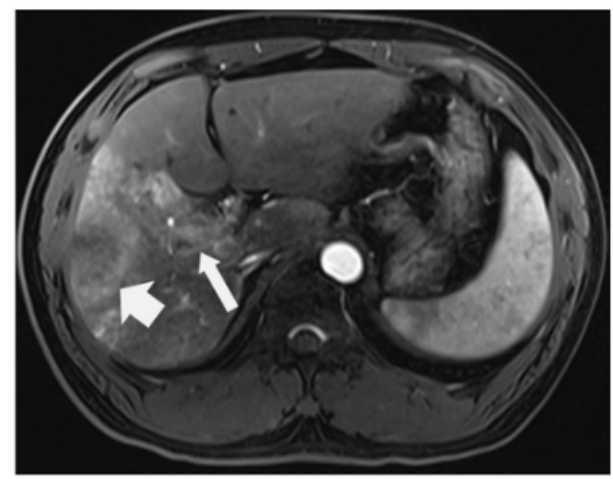

a.

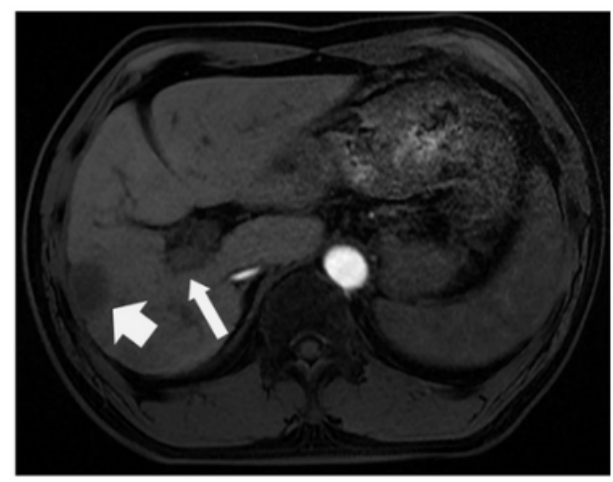

d.

\section{Figure 4}

Images in a 40-year-old man with hepatocellular carcinoma (HCC) with right portal vein tumor thrombus who underwent systemic combination therapy (lenvatinib plus nivolumab) with transarterial chemoembolization (TACE). (a, c) Axial acquired contrast-enhanced T1-weighted MRI and (b) T2weighted MRI at baseline shows $\otimes$ and $\otimes$ segment with irregular masses and right portal vein tumor thrombus (arrow) before treatment. $(d, f)$ Enhanced MRI obtained 3 months after systemic combination therapy with TACE of 3 times shows tumor and the right portal vein thrombus was necrotic and significantly reduced (arrow). (e) Hepatobiliary-phase CT shows lipiodol deposition in the tumor (coarse arrow) and right portal vein thrombus (thin arrow) at 3 months after combination therapy. The patient showed partial response to the combination therapy with TACE 